Archivo Español de Arqueología, 2021, 94, e04

ISSN-L: 0066-6742 | eISSN: 1988-3110

https://doi.org/10.3989/aespa.094.021.04

NOTICIARIO

\title{
Una tabula lusoria hallada en el castellum republicano de Puig Castellar de Biosca en Lleida, Catalunya (180-120 a. C.)*
}

\section{A tabula lusoria found in the Republican castellum of Puig Castellar of Biosca in Lleida, Catalonia (180-120 BCE)}

\author{
Esther Rodrigo Requena \\ Serra Húnter Fellow, Universitat Autònoma de Barcelona \\ esther.rodrigo@uab.cat \\ ORCID iD: https://orcid.org/0000-0003-4771-1216 \\ Núria Romaní Sala \\ Universitat Autònoma de Barcelona - ICAC \\ nuria.romani@uab.cat/nromani@icac.cat \\ ORCID iD: https://orcid.org/0000-0002-5942-6992
}

Enviado: 10-02-2021. Aceptado: 04-03-2021. Publicado online: 30-04-2021

Cómo citar este artículo / Citation: Rodrigo Requena, E. y Romaní Sala, N. (2021). "Una tabula lusoria hallada en el castellum republicano de Puig Castellar de Biosca en Lleida, Catalunya (180-120 a. C.)”. Archivo Español de Arqueología, 94, e04. DOI: https://doi.org/10.3989/aespa.094.021.04

RESUMEN: El presente trabajo pretende dar a conocer el excepcional hallazgo de una tabula lusoria aparecida durante la campaña de 2019 en el castellum republicano de Puig Castellar de Biosca en Lleida. La particularidad de este hallazgo radica en su localización en un complejo militar de la fase inicial de la conquista romana con una cronología muy ajustada que se sitúa entre el 180 y el 120 a. C., y en el hecho de que apareció en su disposición original, en uno de los espacios destinados al alojamiento de la tropa. Estos dos elementos nos permiten afirmar que nos hallamos ante uno de los primeros ejemplos bien datados de un tablero de juego en la península ibérica que nos proporciona pistas sobre la actividad de los soldados en sus momentos de ocio, y nos habla también de la existencia de componentes itálicos entre estos, ya que los juegos sobre tablero serían un pasatiempo desconocido para las poblaciones autóctonas hasta ese momento.

\footnotetext{
Las excavaciones en Puig Castellar de Biosca se inscriben en los proyectos de investigación: "De la consolidación del poder militar romano a la fundación de ciudades (mediados del siglo II a. C.- principios I d. C.) en la cuenca del río Segre: Iesso y Iulia Libica", MINECO/ FEDER, UE (DGYCIT PID2019-104120GB-100-2020-2022) y "Anàlisi arqueològica de la primera ocupació romana a l'altiplà central de Catalunya", Generalitat de Catalunya (CLT009/18/00014).
} 
Palabras clave: ocio romano; tablero de juego; ludus latrunculorum; ejército republicano.

ABSTRACT: We aim to show the exceptional finding of a tabula lusoria that appeared during the 2019 campaign in the Roman Republican castellum of Puig Castellar of Biosca in Lleida. The singularity of this finding lies in its location in a military fortress of the initial phase of the Roman conquest with a very precise chronology that ranges between $180 \mathrm{BCE}$ and $120 \mathrm{BCE}$, and in the fact that it appeared in its original arrangement, in one of the spaces intended for the accommodation of the troops. These two elements allow us to affirm that we are facing one of the first well-dated examples of a game board in the Iberian Peninsula which provides us with clues about the activity of the soldiers in their leisure moments and tells us about the existence of Italic components among them, since board games would be an unknown pastime for the indigenous populations until that moment.

Keywords: Roman leisure; game board; ludus latrunculorum; Roman Republican army.

Copyright: $\odot 2021$ CSIC. Este es un artículo de acceso abierto distribuido bajo los términos de la licencia de uso y distribución Creative Commons Reconocimiento 4.0 Internacional (CC BY 4.0).

\section{INTRODUCCIÓN}

El hallazgo en el año 2019 en el castellum republicano de Puig Castellar de Biosca de una tabula lusoria nos aporta nuevos datos sobre la vida y actividades que realizaban los soldados en su tiempo libre, una vez finalizaban las obligaciones diarias. En cierto modo, nos ofrece una perspectiva más humana de los contingentes destacados en este castellum: nos permite imaginar de manera más vívida su cotidianidad y reconstruir sus actividades habituales, entre ellas el juego.

La particularidad de haberla encontrado en su emplazamiento original y su datación precisa en un enclave militar de los primeros momentos de la conquista romana de Hispania hacen de este hallazgo un elemento único y excepcional. En la península ibérica existen numerosos ejemplos de mesas de juego documentadas tanto en contextos urbanos y rurales, como en campamentos militares, pero en la mayoría de los casos las dataciones se sitúan ya en época imperial, y los hallazgos aparecen como elementos reutilizados o fuera de contexto, sin posibilidad de ofrecer una datación concreta.

\section{CONTEXTO GEOGRÁFICO}

El yacimiento arqueológico de Puig Castellar de Biosca, en la comarca interior de la Segarra, Catalunya (Pera Isern et al., 2016 y 2019), se sitúa en una colina de poca altura formada geológicamente por yesos en su totalidad. Este cerro queda perfectamente protegido por la topografía del entorno por los lados norte, sur y este, y únicamente se puede acceder fácilmente desde la vertiente oeste donde se sitúa el acceso principal (Fig. 1). Desde esta colina se podía controlar fácilmente el paso de la vía que transitaba desde la costa catalana central hasta el valle del Ebro a través del Segre, el afluente más importante que nutre al Ebro, elemento de comunicación clave en el primer siglo de la conquista de Hispania que aseguraba el desplazamiento de tropas hacia el escenario de las guerras celtibéricas, y también su avituallamiento. Podemos afirmar que este asentamiento militar se sitúa en la retaguardia, en un territorio ya relativamente pacificado, pero donde todavía se hacían necesarias las tareas de vigilancia y control sobre la población indígena.

\section{DESCRIPCIÓN DE LA FORTIFICACIÓN}

El enclave ocupa toda la plataforma superior de la colina con una extensión aproximada de 1,6 ha. En la parte central se ubica un edificio perfectamente regular, de planta y arquitectura itálica, que mide $30,2 \times$ $29,7 \mathrm{~m}(100 \times 100$ pies romanos, aproximadamente $)$ y que identificamos como el edificio de comandancia con funciones similares a las de un principia o praetorium, ya que concentraría las funciones de residencia y las actividades administrativas propias del puesto de mando. Este edificio se estructura en torno a un pa- 


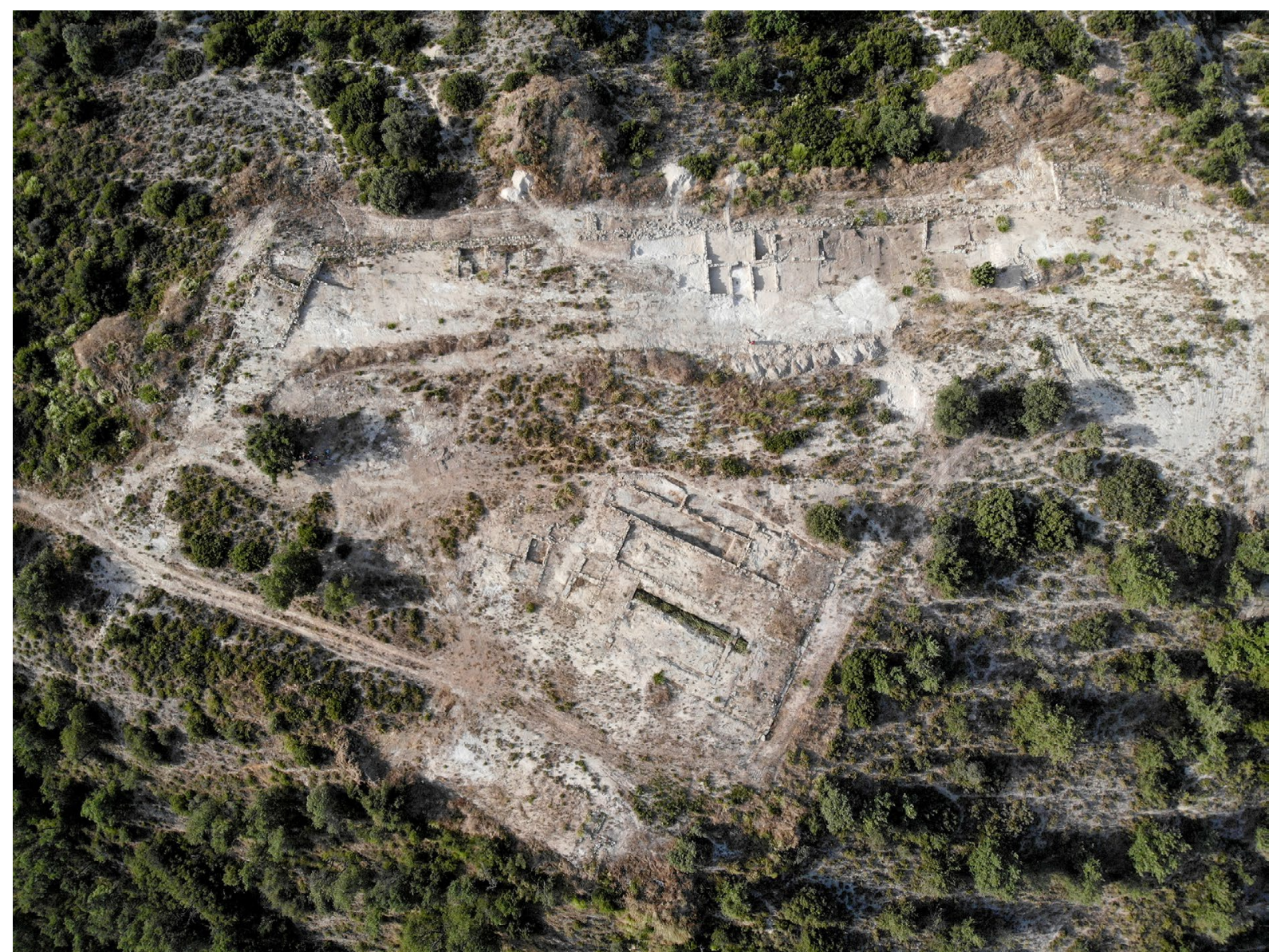

Figura 1. Vista general del yacimiento (fotografía equipo Puig Castellar).

tio central de unos $97 \mathrm{~m}^{2}$ alrededor del cual se sitúan los diferentes ámbitos, que presentan pavimentos de cocciopesto y terrazo y seguramente irían revestidos con decoraciones parietales que se pueden adscribir claramente a los estilos pictóricos dominantes en Italia durante el siglo II a. C. (Romaní et al., 2020). En total se han contabilizado un total de 14 ámbitos de diferentes dimensiones. En el patio se localizó y excavó una cisterna de recogida del agua pluvial con un pozo de extracción, que debía subvenir las necesidades de agua de los ocupantes del edificio central (Fig. 2).

El castellum presenta una muralla, de entre 1 y $1,2 \mathrm{~m}$ de ancho, flanqueada por un total de cinco torres documentadas hasta la fecha, sin foso ni intervallum. La muralla está compuesta por un doble paramento con un relleno de piedra pequeña o emplecton. Los muros exteriores están formados por una base de bloques de piedra arenisca de procedencia local encajados directamente sobre la roca natural tallada, de una altura máxima conservada de $80 \mathrm{~cm}$ de piedra local aportada. i

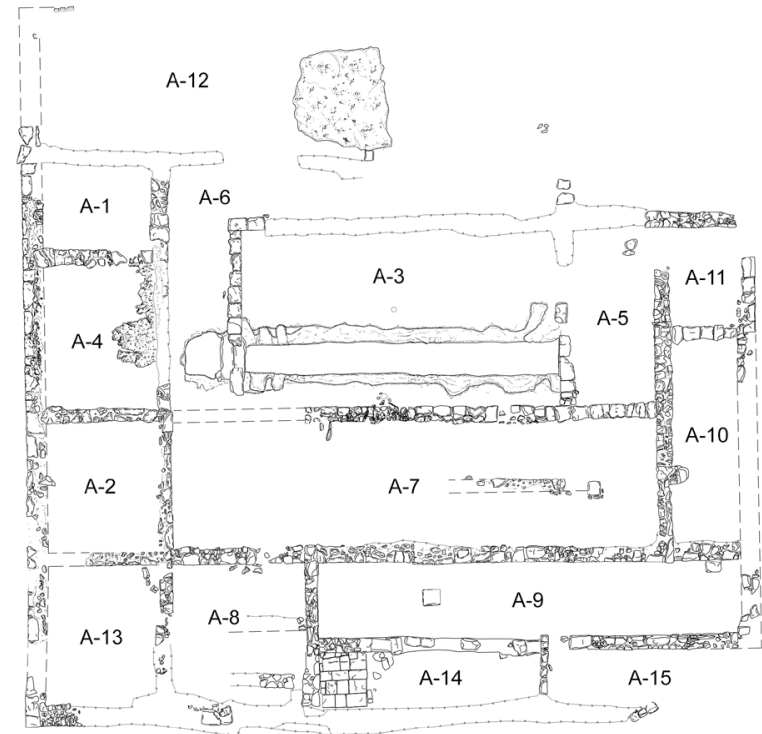

Figura 2. Planta del edificio principal o comandancia (dibujo equipo Puig Castellar). 


\subsection{LAS HABITACIONES ADOSADAS A LA MURALLA}

Adosados a la muralla (Fig. 3) se sitúan los ámbitos presumiblemente ocupados por la tropa; estos espacios se extendían siguiendo todo el perímetro interior del recinto defensivo de manera que la pared de cierre posterior era la propia muralla y, a su vez, servían como refuerzo de su paramento interior. La pendiente de la colina fue nivelada y excavada para poder disponer tanto la muralla, como las construcciones recién descritas. Por el momento, se han localizado ámbitos de este tipo en el lado sur y oeste del complejo.

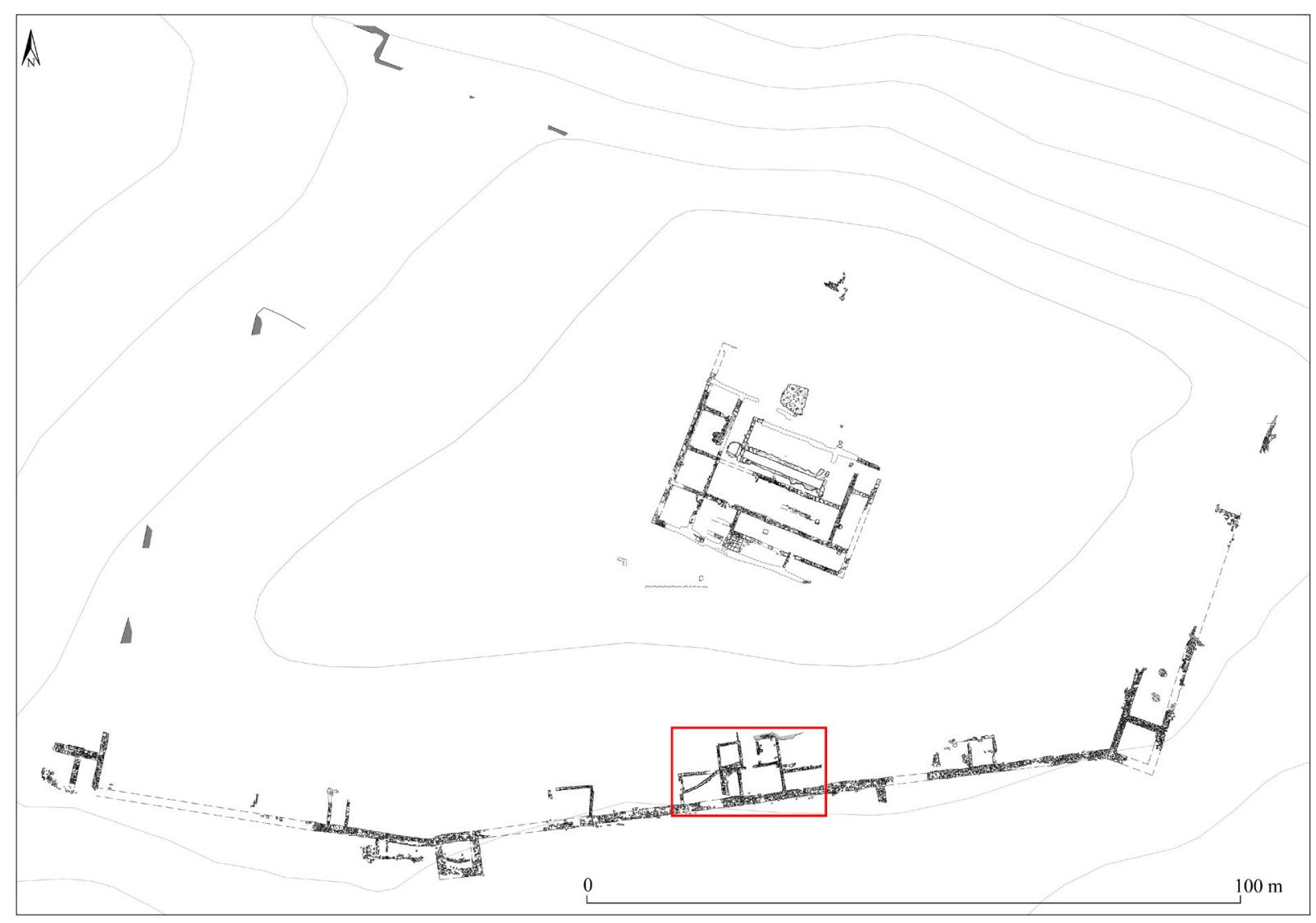

Figura 3. Planta general del castellum. Enmarcadas en rojo, el conjunto de estancias adosadas a la muralla, donde se localizó la tabula lusoria (dibujo equipo Puig Castellar).

Una parte de estos ámbitos se hallan muy arrasados y en algunos puntos del yacimiento han desaparecido prácticamente, conservándose únicamente alguna de las paredes de las habitaciones. Aún así, en los puntos menos erosionados, hemos podido delimitar hasta el momento un total de dieciocho ámbitos. El conjunto que conforman los ámbitos $\mathrm{C} 5$, C6, C7, C8, C9, C10 y C11 (Figs. 3 y 4) que se encuentran adosados al paramento sur de la muralla, son hasta ahora los que presentan un mejor estado de conservación y donde se han podido recoger más datos sobre su momento de uso. Las dimensiones van de los 9,44 $\mathrm{m}^{2}$ de la estancia más reducida a los $27,70 \mathrm{~m}^{2}$ de la más grande. Cada unidad de habitación presenta unas medidas diferentes, seguramente en función de su uso, el espacio disponible y del número de ocupantes.

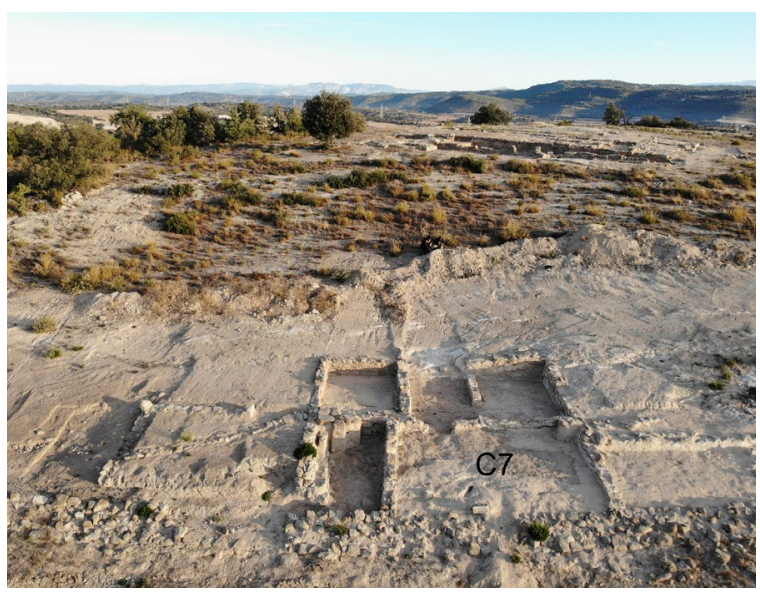

Figura 4. Vista aérea del conjunto de los ámbitos destinados al alojamiento de las tropas; en primer plano el ámbito C7 (fotografía equipo Puig Castellar). 
La técnica constructiva usada en estas estancias consiste en un zócalo de piedra que presenta una altura variable según las necesidades constructivas; las paredes que sirven como refuerzo de las terrazas tienen un mayor alzado, mientras que los otros paramentos presentan una altura conservada inferior. El resto del alzado estaba realizado en tapial o adobe, niveles que hemos podido documentar en los estratos de abandono y derrumbe de las habitaciones. Como en el resto del asentamiento, la mayor parte de la piedra es aportada de algún lugar cercano.

Probablemente, estos habitáculos eran de una sola planta, y se disponían de forma escalonada aprovechando la pendiente de la colina, de manera que se creaban algunos espacios de semisótano, como sería el caso del ámbito C9. Interpretamos que mayoritariamente estos espacios al abrigo de la muralla debían servir como alojamiento de los contingentes acantonados en el castellum, combinando las funciones de un espacio doméstico: dormitorios, almacenaje, espacios de recreo y también de trabajo.

\subsection{El contexto del hallazgo: el Ámbito C7}

El hallazgo que presentamos en este trabajo apareció en el ámbito C7 durante la campaña de 2019. Se trata de una habitación de 6 por 4,30 metros, $25,8 \mathrm{~m}^{2}$ en total, de planta rectangular y que se encuentra adosada a la muralla. Es uno de los ámbitos más grandes y espaciosos de los documentados hasta la fecha. Se entraba a esta habitación por un único acceso que comunicaba con el ámbito C8A, un pasillo-distribuidor que funcionaría como entrada desde el exterior al conjunto de ámbitos que se encuentran en esta zona (Fig. 5).

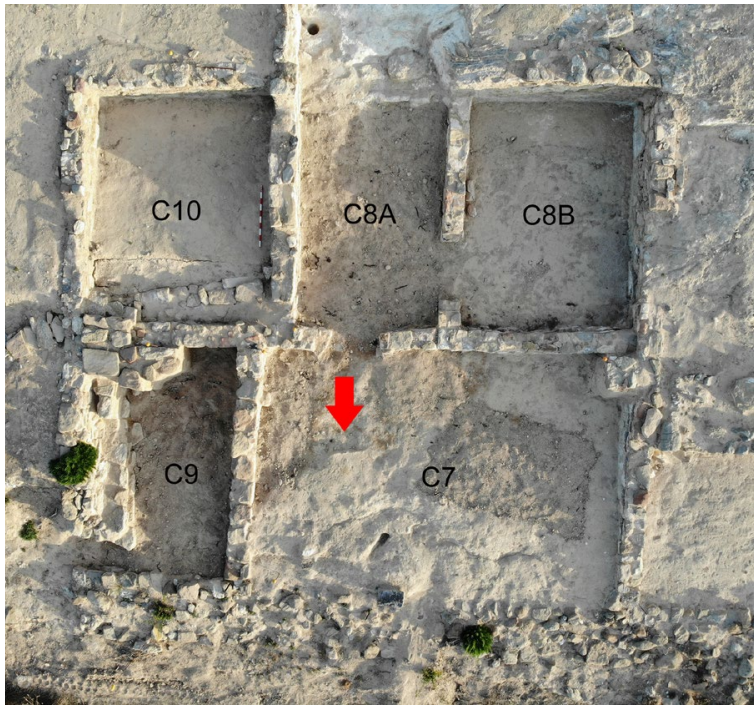

Figura 5. Vista aérea en detalle de las estancias descritas con el lugar de aparición de la tabula lusoria (fotografia equipo Puig Castellar).

La excavación del ámbito C7 permitió documentar los niveles de abandono formados por los restos de los alzados de pared de tapia. Una vez extraídos, quedó al descubierto el nivel de circulación, que consistía en un pavimento de tierra batida; en la parte central de la estancia apareció una estructura de combustión y situado junto a esta, in situ y seguramente en su posición original, la tabula lusoria que nos ocupa. Además de este hogar, al lado del acceso también apareció otra estructura de combustión de menores dimensiones (Fig. 6).

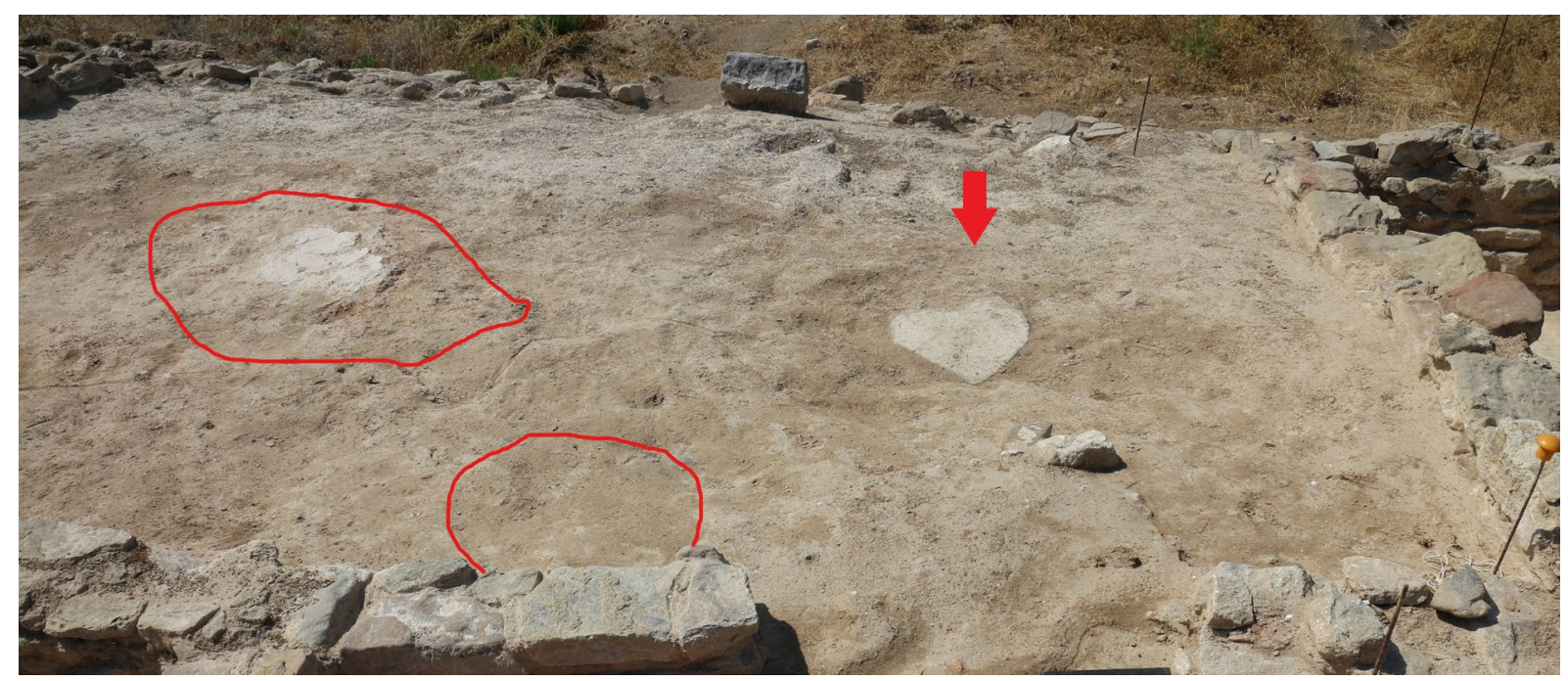

Figura 6. Detalle del ámbito C7 con la tabula lusoria in situ y las estructuras de combustión delimitadas en color rojo (fotografía equipo Puig Castellar). 


\section{LA TABULA LUSORIA DE PUIG CASTELLAR}

Se trata de una losa plana de piedra arenisca de procedencia local, de forma poligonal, de 48,5 $\times 29$ $\times 33 \times 36 \times 26 \mathrm{~cm}$ y entre 5,8 y $7,5 \mathrm{~cm}$ de grosor. $\mathrm{La}$ losa se conserva íntegra pero fracturada en dos partes, que se encontraron articuladas en su posición original. Todo parece indicar que se trataría de una losa que, en origen, estaría tallada de forma rectangular, y que fue reutilizada como tabula lusoria una vez quedó inutilizada para su función primigenia.
En la parte superior de la misma se observaron unas incisiones ${ }^{1}$ que forman una cuadrícula en forma de damero. Determinamos que se trataba de un tablero, una tabula lusoria. Las incisiones no se hallaban grabadas directamente sobre la losa, sino que previamente se había dispuesto una especie de lechada blanca, dispuesta ex professo para poder realizar las incisiones del tablero más fácilmente (Fig. 7).

1 Agradecemos a Francesc Sanahuja su agudeza visual sin la cual las incisiones hubieran pasado desapercibidas.

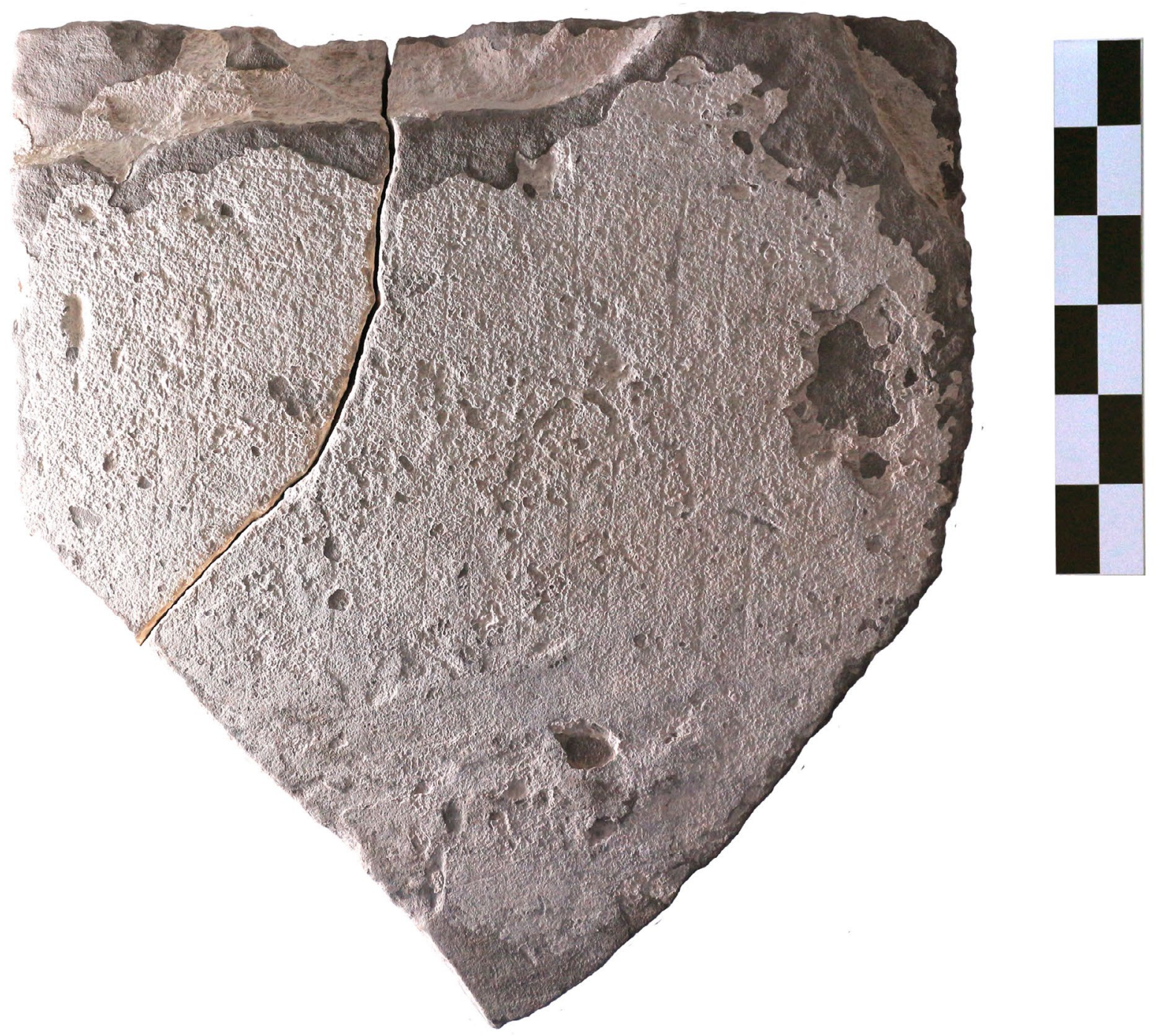

Figura 7. La tabula lusoria tras la restauración (fotografía equipo Puig Castellar). 
El tablero se dispone en la parte más regular y plana de la losa. Presenta una retícula cuadrangular de 11 por 16 casillas, en total 176 casillas. Cada una de las casillas oscila entre los $2 \mathrm{~cm}$ y $2,7 \mathrm{~cm}$ de lado, con tendencia a la forma cuadrada. Las dimensiones de la retícula de juego serían de $37 \times 26 \mathrm{~cm}$. El estado de conservación de la retícula es muy precario debido a la degradación de la piedra, y ha sido necesaria su restauración.
Todo parece indicar que este tablero de juego era de confección casera, probablemente fabricada por los mismos soldados que le dieron uso.

La aparición de esta tabula en su posición original permite reconstruir perfectamente cómo serían los momentos de descanso de los soldados alojados en estas habitaciones. Junto al fuego, debían pasar las horas jugando, con el fin de matar el aburrimiento y los largos ratos de inactividad en un castellum de la retaguardia en que probablemente no había demasiadas distracciones ni acción (Fig. 8).
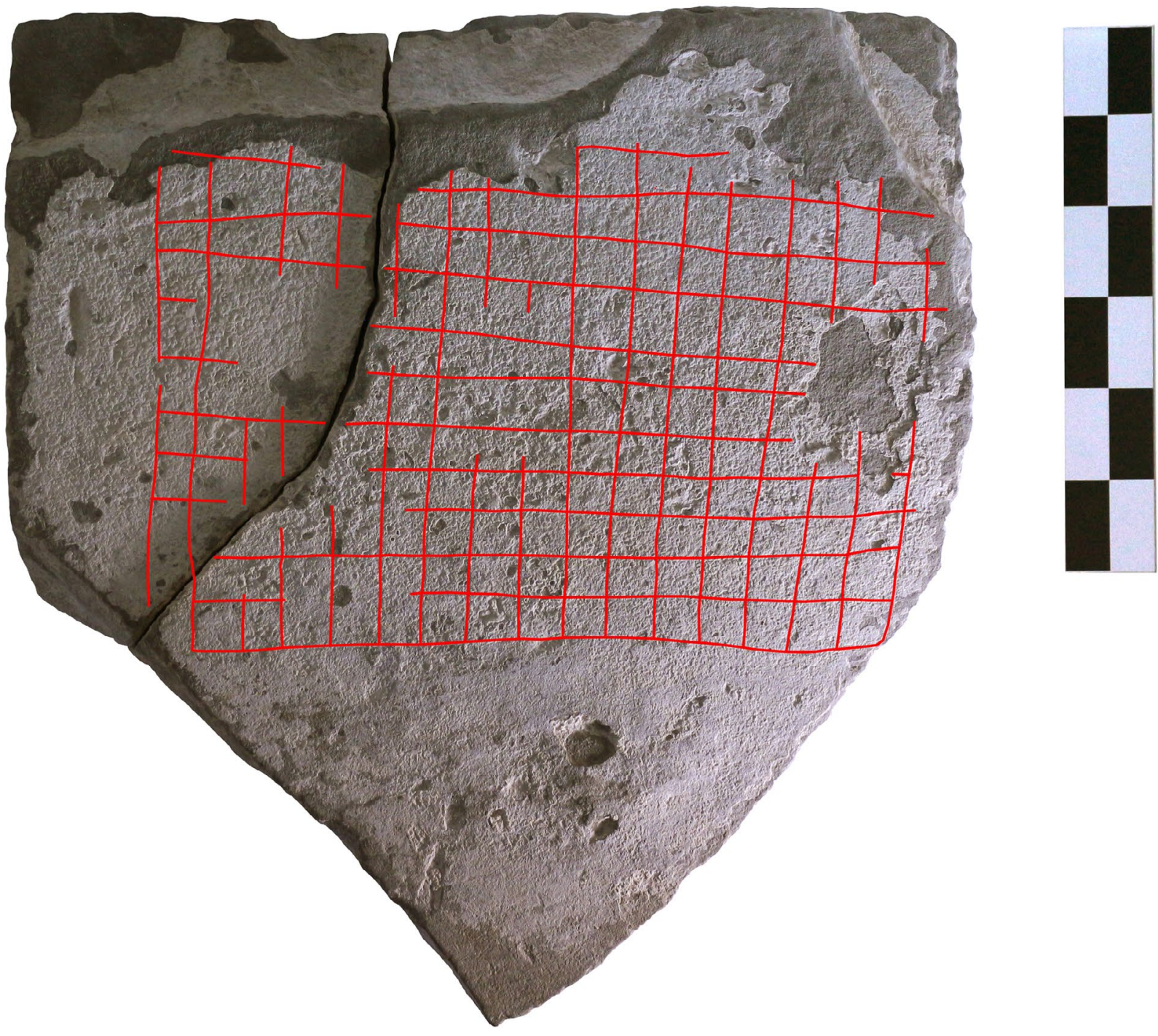

Figura 8. La tabula lusoria con el trazado resaltado para facilitar su visión (fotografía equipo Puig Castellar). 


\section{LAS TABULAE LUSORIAE EN CONTEXTOS MILITARES: TIPOS, SOPORTES Y EJEMPLARES}

Los tableros de juego estaban ampliamente difundidos en el mundo antiguo, con tres grandes grupos de tabulae lusoriae según su trazado: las circulares, las de trazado en forma de retícula y las de forma cuadrangular, pero sin casillas (Jiménez Cano, 2014; Schädler, 2013). La tabula aparecida en el castellum de Puig Castellar pertenece a la segunda categoría, las de trazado reticular.

A lo largo de todo el mundo romano los hallazgos de tableros de juego similares a la tabula de Puig Castellar son numerosos. Los hallamos en contextos urbanos, rurales, incluso en santuarios religiosos (Bendala, 1973; Schädler, 2013, pp. 2842-43 Lambrugo, 2015) y como no, su presencia en yacimientos de carácter militar es más que notable, en sintonía con la pasión de los soldados por el juego que nos relatan las fuentes ${ }^{2}$. Aunque la mayor parte de las tabulae conservadas están elaboradas en materiales perdurables: piedra, cerámica y en casos excepcionales, mármol (Jiménez Cano, 2014, p. 130), es de suponer que la mayoría debían estar fabricadas en madera y no se han preservado (Austin, 1935, p. 80; Jiménez Cano, 2014).

En general, la mayor parte de los tableros de juego con trazado reticular recuperados oscilan entre 8 por 8,7 por 8,9 por 10 , y también 12 por 12 casillas como distribuciones más habituales. Pero también se han encontrado tabulae con dimensiones que superan estas proporciones. Entre estas, destaca el hallazgo en 2006 de una tabula en madera de 17 por 18 casillas que formaba parte del rico ajuar de una tumba de un caudillo germano enrolado como foederatus de las tropas romanas y que se ha fechado hacia el $375 \mathrm{~d}$. $\mathrm{C}^{3}$.

$\mathrm{Si}$ nos centramos en los paralelos procedentes estrictamente de contextos militares, estos son numerosos y aparecen repartidos por todas las provincias romanas. En Britania se documentaron en el campamento de auxilia de Corbridge (Coria), varios fragmentos de tabula lusoria y una retícula de juego incisa en una losa con una trama de 11 por 10 casillas, fechados entre los siglos II-III d. C. (Fig. 9) (Pace, 2015; Austin, 1935). En Vindolanda, se han localizado fragmentos de tableros de juego en forma de casi-

2 Sobre esta afición de los soldados al juego podríamos mencionar sin ir más lejos, el relato evangélico de la crucifixión de Jesús, en que los soldados se jugaron a los dados la capa y la ropa del reo (Jn 19, 23-24; Mt 27,35; Mc 15,24; Lc 23, 34).

3 Hallazgo presentado por Ulrich Schädler en el workshop Tabulae Lusoriae. From Ancient Egypt to Late Antiquity celebrado en mayo de 2019 en la Universidad de Fribourg. lleros (Pace, 2015), así como en Cilurnum (Chesters, Cheshire), y en Richborough (Kent) (Austin, 1935; Pace, 2015), o también, la retícula incisa aparecida en el vallum Adrianum (Jiménez Cano, 2014, p. 130). Todos los ejemplares británicos documentados están realizados sobre piedra. Algunos de estos hallazgos han aparecido acompañados de numerosas fichas de juego (Austin, 1935; Pace, 2015, p. 48).

En la península ibérica se documentan numerosos hallazgos en los campamentos de cronología imperial situados en el cuadrante noroeste. En el campamento de Cidadela (Sobrado dos Monxes, Coruña), activo entre el 123 y el 395 d. C., sede de un contingente de la Cohors I Celtiberorum equitatium civium romanorum adscrita a la Legio VII Gemina, y en el campamento de Aquis Querquennis (Porto Quintela, Ourense), ocupado entre el último cuarto del siglo I d. C. y los primeros decenios del siglo II d. C., se han localizado tableros de juego en forma de retícula incisos sobre tegulae reaprovechadas (Jiménez Cano, 2014, pp. 131-132; Caamaño, 1984).

Los datos más interesantes y abundantes provienen de Petavonium (Rosinos de Vidriales, Zamora), sede de la Legio X Gemina y posteriormente del Ala II Flavia; en este caso, en dos edificios destinados a alojamiento de las tropas, se localizaron varios tableros de juego concentrados en cuatro estancias. Se documentaron una decena de fragmentos de tabulae incisas sobre tegulae (Fig. 9), y numerosas fichas de juego. El elemento más interesante es que las incisiones se habrían realizado antes de la cocción de las tegulae; probablemente se produjeron ex professo algunos tableros de juego dentro de las producciones de material constructivo suministrado al campamento para disfrute de los soldados (Carretero, 1998, pp. 136-137).

\section{EL JUEGO}

Una vez descritas las circunstancias del hallazgo, las características de la tabula y sus paralelos, deberíamos plantearnos para qué juego podría haberse empleado. Sin duda alguna, se trataría de un juego destinado a adultos, con lo cual podemos descartar los juegos infantiles (Salza Prina Ricotti, 1995; Fittà, 1997; Dasen y Schädler, 2013; Dasen, 2018).

A partir de las fuentes textuales y los hallazgos arqueológicos conservados, sabemos que los romanos eran muy aficionados a todo tipo de juegos (Dasen, 2018, p. 25; Guidi, 2015, pp. 125-150): como los de azar, los de estrategia y, sobre todo, a cualquier juego donde se pudiera apostar (Quintana 2009; Jiménez Cano, 2014, p. 27). Los dos juegos de los que tenemos noticia a través de las fuentes y que podían encajar con las características de nuestra tabula lusoria podrían ser, o 

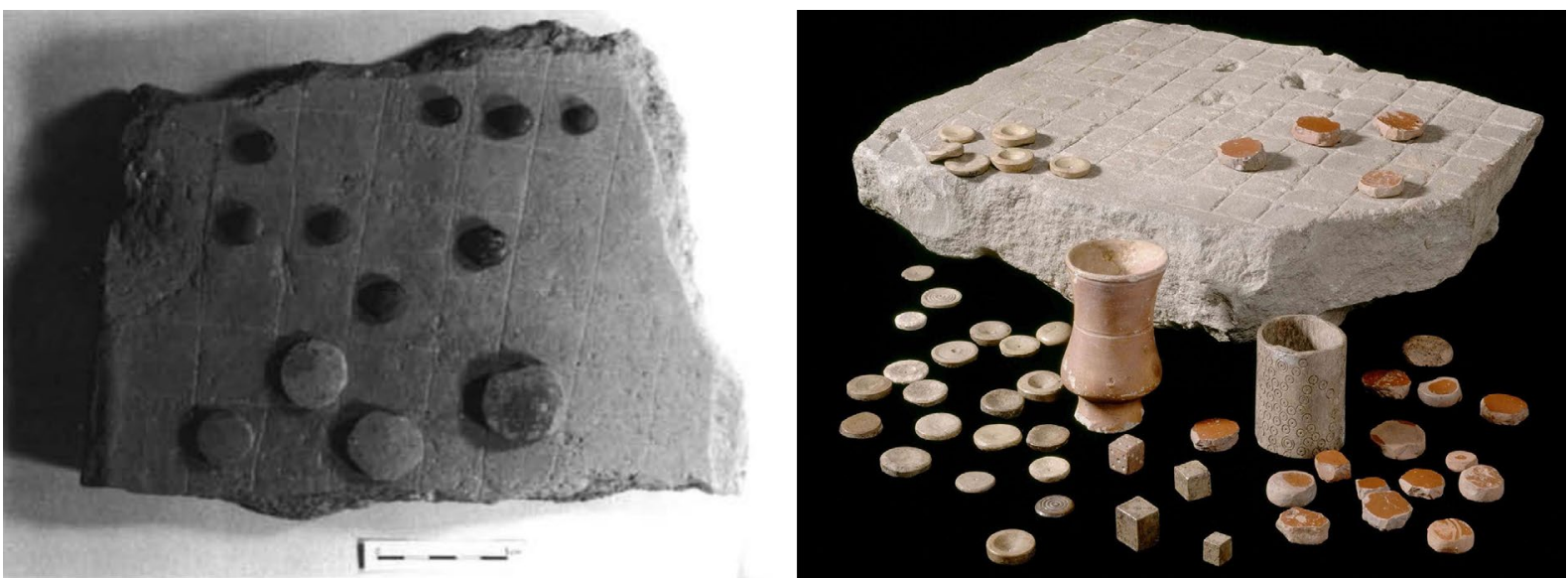

Figura 9. Fragmento de tablero hallado en Petavonium (fotografía Carretero Vaquero, 1998, lám. I) y tabula lusoria de Corbridge (fotografía Historic England Archive Photo Library ref: J970223).

bien, el juego de los calculi, o bien el juego conocido como ludus latrunculorum, o juego de los mercenarios (Austin, 1935; Bell, 1979, pp. 84-87; Schädler, 2013).

La afición desmedida por el juego en general y sobre todo por los juegos de azar, provocó que a finales de la República se tuvieran que promulgar diversas leyes, entre las que destacan las leges Aleariae (Quintana, 2009; Jiménez Cano, 2014, p. 127) que prohibían los juegos de azar, y especialmente las apuestas; en el caso del ludus latrunculorum, al tratarse de un juego de estrategia, con un cierto prestigio entre los mejores jugadores (Quintana, 2009, pp. 17-18) probablemente no quedaba afectado por estas leyes, aunque sí la posibilidad de establecer apuestas.

En todos los ejemplos aparecidos en asentamientos militares, mayoritariamente se ha interpretado que estos tableros se habrían usado para el juego del ludus latrunculorum, al tratarse de un juego de estrategia y táctica militar.

Las fuentes literarias nos proporcionan algunas referencias de este juego y de sus características. La primera mención del juego del ludus latrunculorum aparece en Marco Terencio Varrón, escritor y militar de finales de la República, quien en su obra De Lingua Latina, 10, 22, comparaba las tablas de declinaciones latinas con la cuadrícula del ludus latrunculorum.

Posteriormente, algunos poetas mencionan el latrunculi como metáfora del combate y estrategia amorosa en el caso de Ovidio ${ }^{4}$, y como parte de las pullas burlescas en el caso de Marcial ${ }^{5}$. La mención más im-

4 Ovidio, Ars Am., 2. 203-208. También en las Tristia encontraremos algunas referencias en Tristium 2. 471-484.

5 Marcial en algunos de sus epigramas: en el Libro 7, el epigrama LXXII, y también en el libro 14, el epigrama XX. portante aparece en un panegírico anónimo en verso conocido como Laus Pisonis; en este poema, dedicado a uno de los miembros de la familia Pisón, aparecen descritos algunos de los movimientos y estrategias del ludus latrunculorum (Richmond, 1994).

También destaca Julio Pólux, gramático griego, que entre el 166-176 d. C. compuso un gran tratado léxico, el Onomasticon, y en donde describe el latrunculi (Salza Prina Ricotti, 1995, pp. 13-15 y 109-110; Dasen, 2018, p. 28).

La última mención relevante aparece en Macrobio, que posiblemente vivió a caballo de los siglos IV y V d. C.; en sus Saturnalia I.5.11, el autor lamenta la falta de interés general por mantener discusiones profundas y la preferencia por perder el tiempo con juegos como el latrunculi. Después de esta última referencia, el juego del ludus latrunculorum ya no volverá a ser mencionado, y al parecer, cayó en el olvido.

Sobre el reglamento y desarrollo del juego, los intentos de reconstrucción a partir de las menciones documentales y la arqueología han sido numerosos sin que se hayan podido llegar a establecer con total certeza ni las reglas ni su desarrollo. Los especialistas se muestran de acuerdo en que sobre el tablero dos jugadores confrontaban dos ejércitos, y se erigía como ganador el que conseguía mantener un mayor número de piezas sin capturar en juego. La estrategia consistiría en intentar acorralar, inmovilizar y capturar las fichas del contrincante hasta conseguir su rendición ${ }^{6}$.

6 Sobre el desarrollo del juego del ludus latronculorum: Austin, 1935; Bell, 1979, pp. 84-87; Richmond, 1994; Salza Prina Ricotti, 1995, pp. 102-107; Fittà, 1997, pp. 167-171 y especialmente Schädler, 2001 y 2013. 


\section{LA DATACIÓN}

El período en que estuvo en activo el castellum fue bastante breve: a partir de los materiales documentados en las excavaciones que se llevan a cabo desde el año 2012, se constata que probablemente estuvo ocupado únicamente entre el 180 a. C. y el 120 a. C. La vajilla fina de importación está copada por la presencia de producciones del grupo de la campaniense de tipo A (224 ind.) donde predomina la presencia de formas de la variedad media (Lamboglia 5, 6, 27a-b, 27c, 28 a-b, 31 a y b, 33 a y b, 34, 36 y F3130); cabe destacar la presencia de algunas formas antiguas del repertorio de la campaniense A, como son el plato Lamboglia 23 (15 ind.), fragmentos de diferentes gutti F8150, y de tres copitas del Taller de Roses, de la forma $10 \mathrm{~A}$, que nos llevan a proponer una cronología inicial para el establecimiento entorno al 180 a. C. Asimismo cabe destacar la escasa presencia de campaniense de tipo B (9 ind.) con formas de producción calena media Lamboglia 2, 3 y Morel F4753, que podemos atribuir al último cuarto del siglo II a. C. Por lo que se refiere a la cerámica común y de cocina, se detecta la presencia de vasos de paredes finas, formas Mayet II y III, mayoritariamente de origen local y, puntualmente, de factura itálica. Dentro de la vajilla de mesa, predominan las producciones oxidadas tanto romanas como de tradición ibérica, así como algunas ollas de cerámica reducida de cocina, estas últimas muy poco representadas. Destacamos la presencia de cerámica ibérica gris (29 ind.) e ibérica pintada (83 ind.) y algunas importaciones de cazuelas, patinae y morteros de origen itálico (formas Emporiae 36.2 y COM-ITA 8, variantes a, c, d y e), junto a una mínima representación de cazuelas púnicas (4 ind.). Las formas de ánfora documentadas son las habituales de este período: destacamos las ánforas vinarias greco-itálicas (clásicas y de transición) y Dressel 1A, de procedencia itálica diversa, y las ánforas olearias Apani I-Giancola de la Apulia, que marcan una horquilla cronológica entre 175 a. C. y 120 a. C. El último grupo identificado son las ánforas de origen africano, seis formas T.7.4.1.1 y dos T.7.4.2.1, producción que tendría su momento álgido entre mediados y el tercer cuarto de siglo II a. C. Estas importaciones conviven con una gran cantidad de envases ibéricos de boca plana de origen local y regional (40\% de los ejemplares). A la vista del material documentado todo parece indicar que alrededor del 120 a. C. se abandonaría el yacimiento de Puig Castellar (Pera Isern et al., en prensa).

Esta datación en una franja tan concreta y precisa hace más valioso aún si cabe el hallazgo de la tabula lusoria. La disposición de tablero de juego sobre la última pavimentación de la estancia y cubierta por los niveles de derrumbe de la habitación nos hace pensar que estuvo en uso hasta el momento final de ocupación del castellum. De esta manera, podemos suponer un periodo amplio de utilización que debería situarse entre mediados del siglo II a. C. y su abandono. Todos los casos documentados hasta la fecha, son enclaves militares con una cronología que se sitúa ya en época imperial, en un momento avanzado del siglo I d. C. y, en algunos casos, llegando hasta los siglos III-IV d. C. Ninguno de estos hallazgos ha aparecido en un contexto estratigráfico definido que permita una datación exacta, y para la mayor parte de los ejemplos localizados tampoco tenemos definido el contexto espacial de su localización de una forma precisa, con la única excepción de Petavonium.

\section{CONCLUSIONES}

Esta tabula constituye el testimonio más antiguo de la práctica de juegos de mesa, y en concreto del juego del ludus latronculorum, en un contexto militar romano hispánico, ya que el castellum de Puig Castellar de Biosca es, a fecha de hoy, uno de los primeros enclaves militares estables que podemos situar en Hispania en el momento inicial de la conquista romana tras la II Guerra Púnica, situado en una de las vías de penetración hacia el valle del Ebro y la Celtiberia, escenario de los conflictos bélicos durante buena parte de este primer siglo de ocupación romana. Asimismo, el castellum de Puig Castellar de Biosca se erige como uno de los primeros ejemplos en el ámbito romano de un asentamiento militar estable con una serie de características y atributos que pueden ayudar a definir cómo sería la arquitectura y la distribución de los establecimientos militares republicanos (Fig. 10).

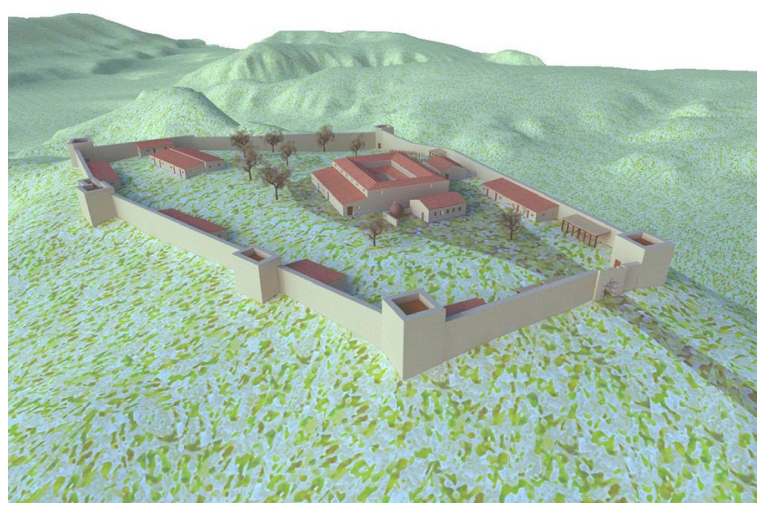

Figura 10. Propuesta de reconstrucción 3D del castellum (infografía Jordi Barriuso). 


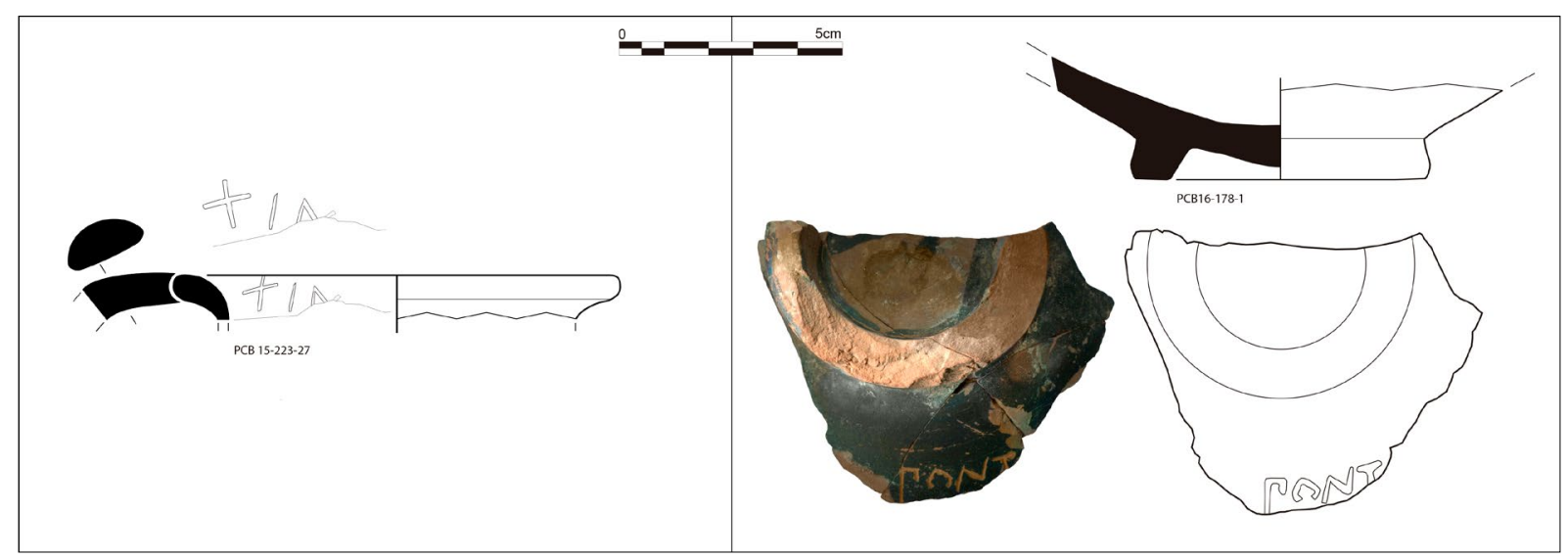

Figura 11. Grafitti ibérico y grafitti en latín hallados en Puig Castellar (dibujos y fotografía equipo Puig Castellar).

Proponemos que este tablero de juego habría servido presumiblemente para jugar al ludus latronculorum; en contra de esta hipótesis se puede esgrimir el hecho que las medidas del damero no se corresponden con las medidas usuales documentadas y consideradas como "canónicas" para este juego. En ese sentido cabe decir que a medida que aumentan los hallazgos, van apareciendo tabulae que presentan un número de casillas que se aleja bastante de los modelos que habían sido considerados como los usuales, y que se proponen como variantes locales (Pace, 2015, p. 47).

En nuestro caso, no se ha encontrado ninguna ficha ni ningún elemento relacionado con la práctica del juego que contribuya a su identificación; posiblemente en el momento en que se abandonó el campamento, los soldados debían llevarse en su equipaje los elementos fácilmente transportables como las fichas de juego, dejando atrás el tablero pesado e incómodo de transportar.

El hecho de que hayamos encontrado una tabula en un contexto militar de un momento tan inicial de la presencia romana en Hispania nos proporciona pistas sobre la composición de las fuerzas desplegadas en territorio hispano y refuerza la hipótesis de que una parte de los contingentes provenía de la península itálica, ya que en el mundo indígena local no tenemos constancia de que estos juegos fueran conocidos ni practicados ${ }^{7}$. Así pues, podemos pensar en una tropa formada por itálicos e indígenas; un elemento que refuerza esta propuesta es el hallazgo de un grafito en latín, grabado en el pie de una copa de barniz negro del grupo de la Campaniense A, cuya lectura sería PONT [--], y podría tratarse de un cognomen latino. Los rasgos paleográficos son propios

\footnotetext{
7 No hemos encontrado ningún paralelo en contexto ibérico.
}

de la época republicana, y encajan perfectamente con la cronología general del yacimiento ${ }^{8}$; a este hallazgo habría que sumar un grafito inciso sobre un borde de una jarrita de cerámica gris ibérica, en alfabeto ibérico, en que se puede leer TABAN, posiblemente el nombre del propietario de la jarrita. Ambos epígrafes sobre cerámica constituyen el testimonio de esta diversidad de procedencias y filiaciones (Fig. 11).

De hecho, debemos entender el papel que jugarán estos enclaves militares y las tropas de auxiliares no solo como una herramienta de control y dominio de los nuevos territorios incorporados a Roma, sino también como un factor de aculturación y de introducción de las costumbres romanas entre las poblaciones indígenas. Sin duda, la práctica de los juegos de azar y de estrategia formaría parte de estos mores romanorum que pronto serán adoptados y convertidos en un elemento más del entretenimiento de las poblaciones asimiladas.

\section{BIBLIOGRAFÍA}

Austin, R. G. (1935). "Roman Board Games II". Greece \& Rome, 4.11 (feb. 1935), pp. 76-82.

Bell, C. (1979). Board and Table Games from many Civilizations, vol. 1 y 2. London: Dover Publications.

Bendala Galán, M. (1973). "Tablas de juego en Itálica”. Habis, 4, pp. 263-272.

Caamaño Gesto, J. M. (1984). "La cohors I Celtiberorum y su campamento de Cidadela (Sobrado dos Monxes-Coruña)". Cuadernos de Estudios Gallegos, XXXV, pp. 71-79.

Carretero Vaquero, S. (1998). "El Ludus latrunculorum, un juego de estrategia practicado por los equites del Ala Flavia II'. Boletín SAA, 64, pp. 117-140.

8 Debemos a la inestimable ayuda de Isabel Rodà, catedrática emérita de Arqueología de la UAB, la lectura y descripción paleográfica de este grafito. 
Dasen, V. (2018). "Histoire et Archéologie de la culture ludique dans le monde gréco-romain. Qüestions Méthodologiques". Kentron, 34, pp. 23-50.

Dasen, V. y Schädler, U. (Coords.) (2013). "Dossier Jeux et jouets gréco-romaine". Archéothéma. Histoire et archéologie, 31 (nov.-déc. 2013).

Fittà, M. (1997). Giochi e giocattoli nell'Antichità. Milano: Leonardo Arte.

Guidi, F. (2015). Vacanze Romane. Tempo libero e vita quotidiana nell'antica Roma. Milano: Mondadori Libri.

Jiménez Cano, C. (2014). "Estudio preliminar sobre los juegos de mesa en Hispania". Antesteria, 3, pp. 125-138.

Lambrugo, C. (2015). "Giocare per terra...e per mare!". En: Lambrugo, C. y Slavazzi, F. eds., I materiali della Collezione Archeologica "Giulio Sambon" di Milano 1. Tra alea e agòn: giochi di abilità e di azzardo. Milano: All'Insegna del Giglio s.a.s., pp. 25-30.

Pace, A. (2015). "Miles ludens. Il gioco e i soldati nella Britannia romana". En: Lambrugo, C. y Slavazzi, F., eds., I materiali della Collezione Archeologica "Giulio Sambon" di Milano 1. Tra alea e agòn: giochi di abilità e di azzardo. Milano: All'Insegna del Giglio s.a.s., pp. 43-49.

Pera Isern, J., Carreras Montfort, C., Romaní Sala, N., Rodrigo Requena, E., Padrós Font, N. y de Solà Gómez, G. (2016). "El proceso de implantación territorial romana en el NE de la Provincia Citerior en el siglo II a.C.". En: Pera, J. y Vidal, J., eds., Fortificaciones y Control del Territorio en la Hispania Republicana. Zaragoza: Pórtico, pp. 167-205.

Pera Isern, J., Rodrigo Requena, E., Romaní Sala, N. y Carreras Montfort, C. (2019). "Puig Castellar de Biosca (Lleida). Una fortificación romana del siglo II a.C. en el noreste de la Hispania Citerior". Gladius, 39, pp. 19-43.
Pera Isern, J., Rodrigo Requena, E., Romaní Sala, N., Carreras Montfort, C. y Catarineu Iglesias, L. (en prensa). "El contexto cerámico del castellum republicano de Puig Castellar de Biosca (180-120 a.C.)". En: Actas del $V$ Congreso Internacional de la Sociedad de Estudios de la cerámica antigua en Hispania celebrado en Alcalá de Henares (6-8 noviembre de 2019).

Quintana Orive, E. (2009). "D.11.5 (De aleatoribus) y C.3.43 (De aleae lusu et aleatoribus): Precedentes romanos del contrato de Juego". Anuario Jurídico y Económico Escurialense, XLII, pp. 17-37.

Richmond, J. (1994). "The Ludus Latrunculorum and Laus Pisonis 190-208". Museum Helveticum, 51.3, pp. 164-179. http://www.jstor.com/stable/24818294.

Romaní Sala, N., Rodrigo Requena, E., Pera Isern, J. y Gutiérrez Garcia-Moreno, A. (2020). "El material constructiu de la fortificació militar de s. II a.C. de Puig Castellar de Biosca (Lleida): Paviments, revestiments parietals i cobertes”. En: Aquilué, X., Beltrán de Heredia, J., Caixal, À., Fierro, J. y Kirchner, H., eds., Estudis sobre ceràmica i arqueologia de l'arquitectura. Homenatge al Dr. Alberto López Mullor. Barcelona: Diputació de Barcelona, MUHBA, MAC, UAB, pp. 391-403.

Salza Prina Ricotti, E. (1995). Giochi e giocattoli. Vita e costumi dei Romani Antichi 18. Roma: Edizioni Quasar.

Schädler, U. (2001). "A forgotten Roman Game of Strategy reconstructed". Abstract Games, 7, pp. 10-11.

Schädler, U. (2013). "Games, Greek and Roman”. En: Bagnall, R. G., Brodersen, K. Champion, C. B., Erskine, A. y Huebner, S. R. (Eds.). The Encyclopedia of Ancient History. London: Blackwell Publishing Ltd, pp. 28412844. 